Supplementary Information for

\title{
Unexpected effects of catalytic amounts of additives on crystallization from the gas phase: Depression of the sublimation temperature and polymorph control
}

\author{
Naghmeh Kamali, Andrea Erxleben*, and Patrick McArdle* \\ School of Chemistry, National University of Ireland, Galway, Ireland
}

\section{Sublimation apparatus.}

The sublimation apparatus has been described previously. ${ }^{1}$ It consists of a small heater placed under a Pyrex Petri dish. The Petri dish lid acted as a condensing plate (Figure S1). The sample (100-500 mg) was placed directly over the heater. The sublimation apparatus was operated inside a vacuum oven. The vacuum in the oven was determined to be $0.2 \mathrm{~mm} \mathrm{Hg}$ using an Edwards Pirani 501 vacuum gauge for all experiments other than the PABA sublimation carried out at $140{ }^{\circ} \mathrm{C}$ and $400 \mathrm{~mm} \mathrm{Hg}$. The pressure in this case was measured using the vacuum gauge on the vacuum oven. The double sided sticky-tape was attached to the underside of the Petri dish lid. Thermocouples and Omron E5CSV temperature control units were used to monitor the temperatures of the sample and the condenser plate and an ISO-TEC IPS 303A power supply was used to operate the heater, Figure S2. The temperatures quoted are believed to be accurate to better than $\pm 1.0^{\circ} \mathrm{C}$. The temperature of the condenser plate was approximately $20^{\circ} \mathrm{C}$ lower than the heated sample. All sublimations were carried out below the melting points of the compounds involved. The melting points are CBZ 190-192, piracetam 162, diflunisal 210-211 and paminobenzoic acid $187^{\circ} \mathrm{C}$.

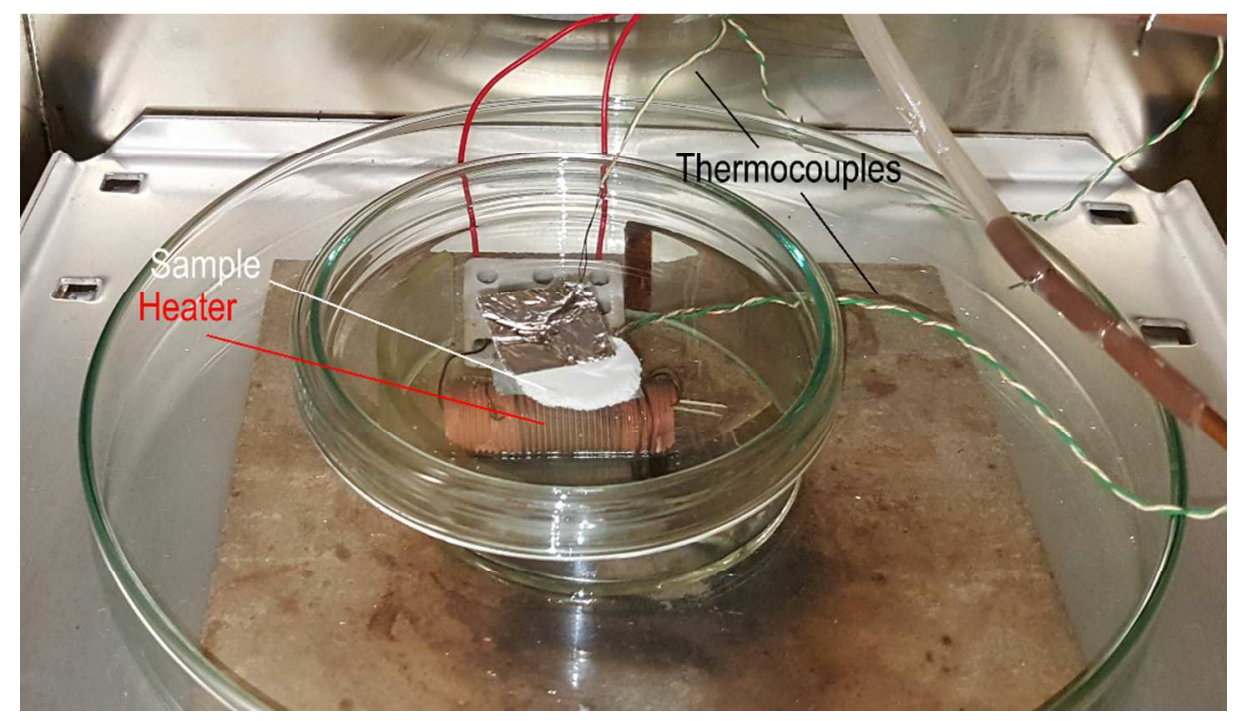

Figure S1. Sublimation apparatus inside vacuum oven. 




Figure S2 Thermocouple controllers and the heater power supply. 


\section{Molecular orbital calculations.}

Molecular orbital calculations were carried out using the Firefly program (accessed 6-Jan-2016) which was driven by Moilin within the Oscail package. ${ }^{2,3}$ A DFT calculation was used to optimize the structures of the CBZ-acteamide adduct, the CBZ H-bonded dimer and their components using the B97D dispersion corrected functional and a 6-31G* basis set, Table S1. The energies of the adducts, less the energies of their components, are shown as $\Delta \mathrm{E}$.

\begin{tabular}{|l|l|l|}
\hline \multicolumn{3}{|c|}{ Table S1 Calculated energies } \\
\hline Structure & \multicolumn{1}{|c|}{ Energy $/ \mathrm{kJmol}^{-1}$} & $\Delta \mathrm{E} / \mathrm{kJmol}^{-1}$ \\
\hline Acetamide & -548966 & \\
\hline Carbamazepine (CBZ) & -2003349 & \\
\hline CBZ-acetamide & -2552389 & -74 \\
\hline CBZ dimer & -4006771 & -72 \\
\hline
\end{tabular}

\section{Hydrogen-bonding and dispersion forces in the structure of PABA forms I and V}

The crystal structures of PABA forms I and V both have very short $b$ axes of just $3.73 \AA$ and they have closely related structures. Both structures consist of relatively flat hydrogen-bonded zigzag chains which are stacked along the $b$ axis, Figure S3. ${ }^{4}$.

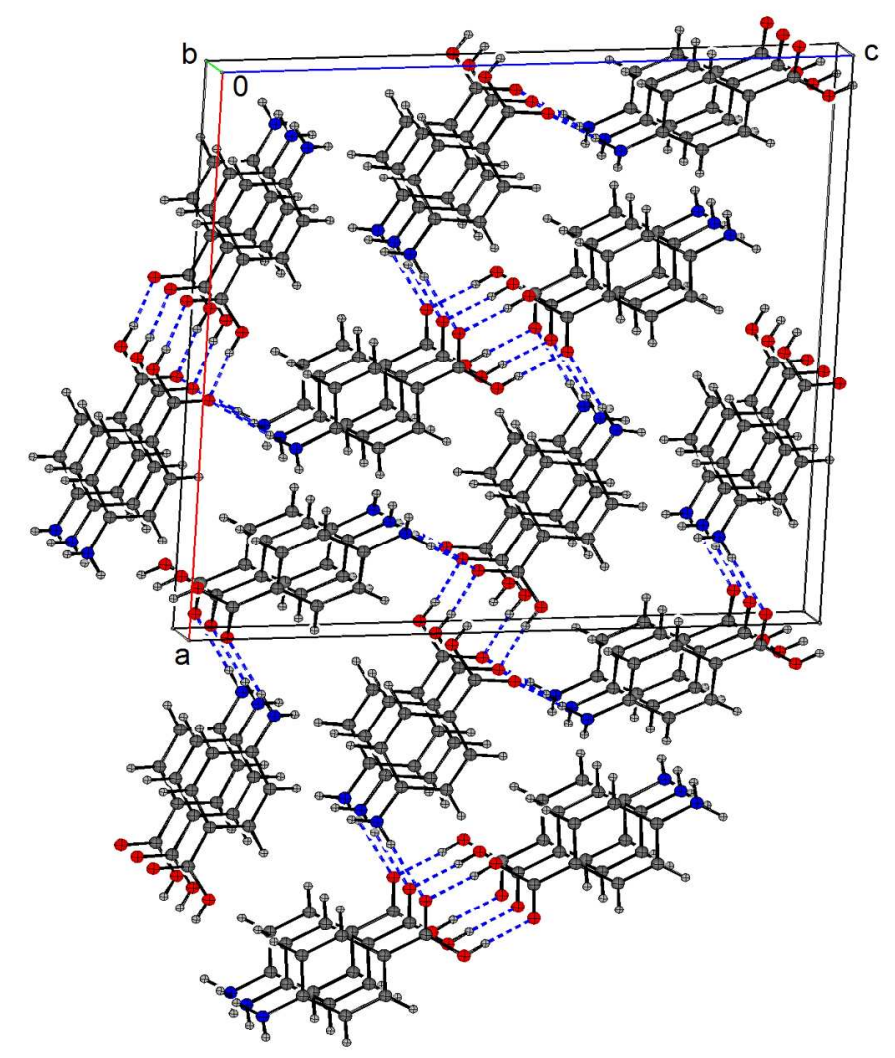

(a) 


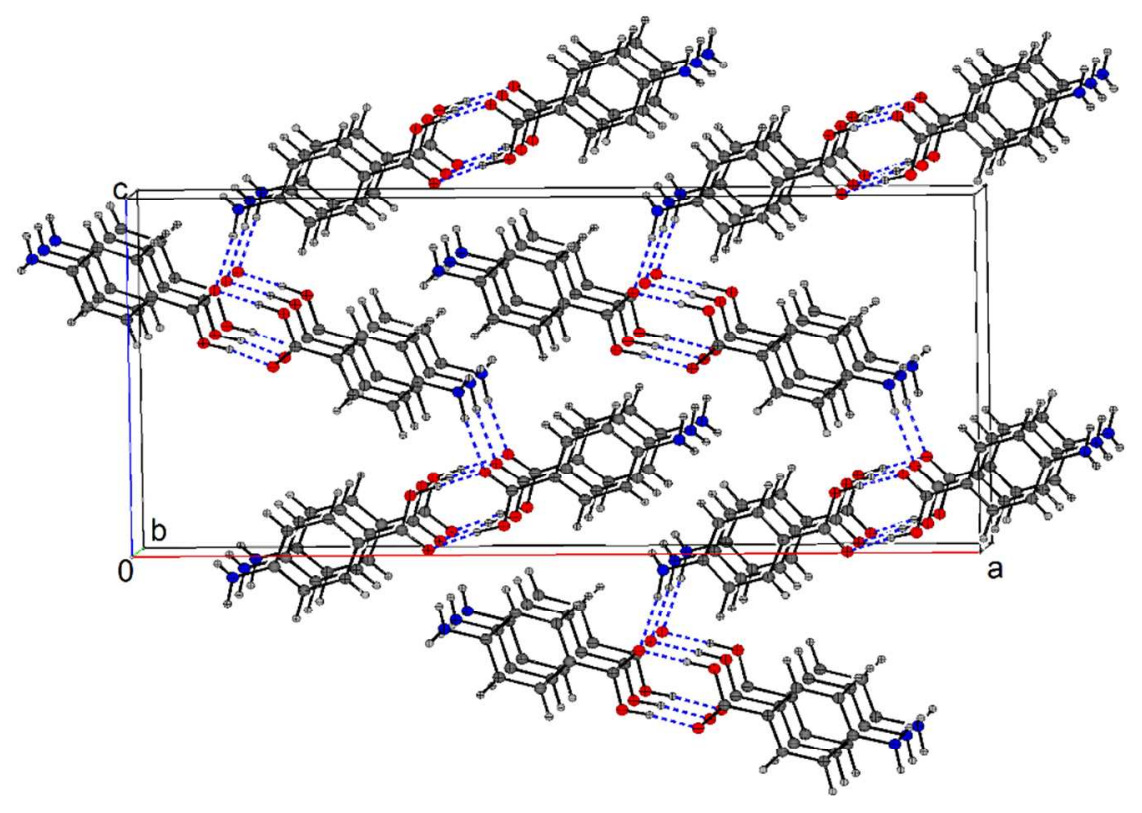

(b)

Figure S3. The crystal packing of PABA (a) form I and (b) form V. The hydrogen bonds are shown as blue dashed lines.

The structures differ in that there is a centre of symmetry in form 1 (space group $P 2_{1} / c$ ) which is not present in form $\mathrm{V}$ (space group Pna2 $2_{1}$ ). In both structures a large fraction of the atoms in each molecule are in vdW contact with their neighbors above and below them in the stacks ( 81 and $78 \%$ respectively). It has been shown that the magnitude of the dispersion force between molecules is related to this fraction. ${ }^{5}$ 


\section{FT-IR spectra of PABA forms I and V}

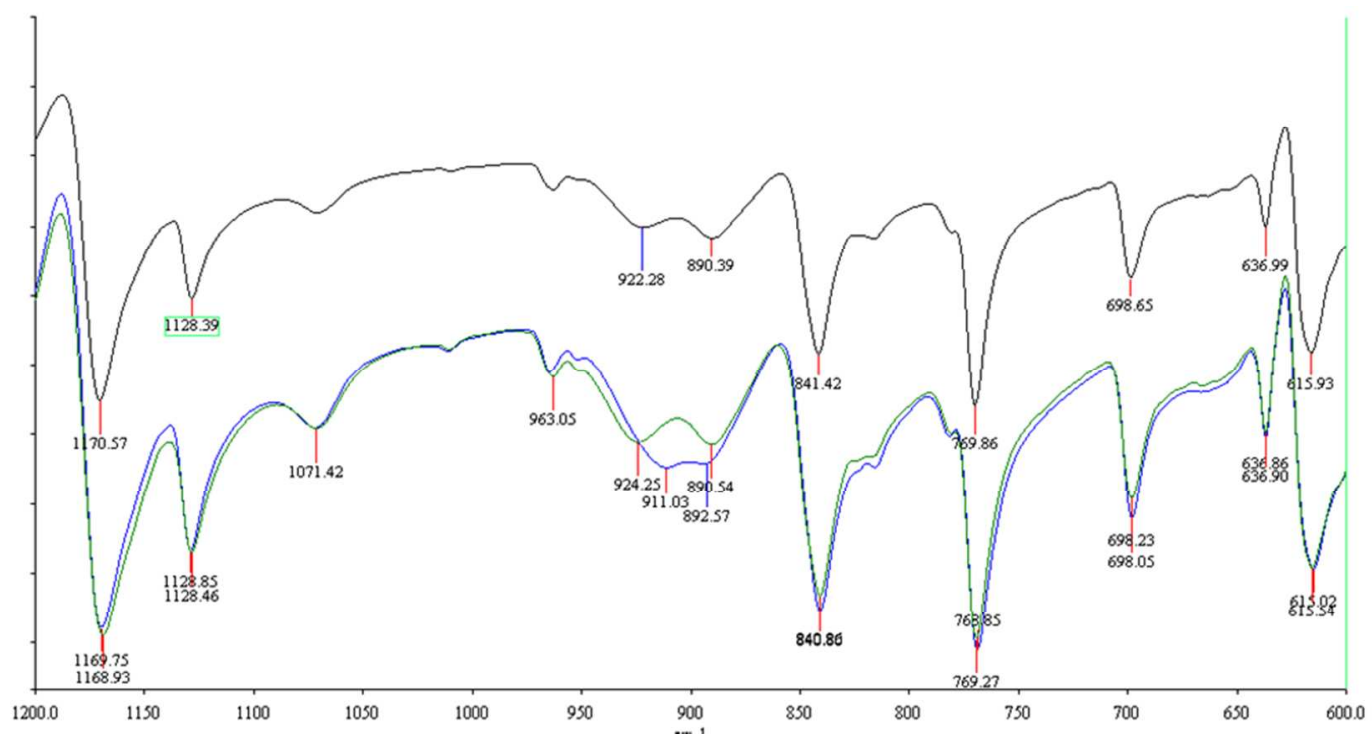

Figure S4. FT-IR spectra of PABA form I (black), PABA form V (blue) and PABA sublimed onto a form I template at $140{ }^{\circ} \mathrm{C}$ (green).

The only significant difference in the FT-IR spectra of PABA forms I and V is in the peak close to $920 \mathrm{~cm}^{-1}$ in form 1 which is shifted by $11 \mathrm{~cm}^{-1}$ in form 5 .

(1) Karpinska, J.; Erxleben, A.; McArdle, P., Cryst. Growth Des. 2013, 13, 1122-1130.

(2) Granovsky, A. A. Firefly (previously PC GAMESS) 2016.

http://classic.chem.msu.su/gran/firefly/index.html

(3) McArdle, P.; Hu, Y.; Lyons, A.; Dark, R., CrystEngComm 2010, 12, 3119-3125.

(4) Benali-Cherif, R.; Takouachet, R.; Bendeif, E.-E.; Benali-Cherif, N., Acta Cryst. C 2014, 70, $323-$ 325.

(5) Walshe, N.; Crushell, M.; Karpinska, J.; Erxleben, A.; McArdle, P., Cryst. Growth Des. 2015, 15, 3235-3248. 